\title{
Coordinated Control Mechanism for Voltage Stability Utilizing Aggregation of Reactive Power Compensation Techniques
}

\author{
Jibran Ali \\ DITEN \\ University of Genova \\ Genova, Italy \\ jibranali.mean4sg@gmail.com
}

\begin{abstract}
Reactive power provision is an essential ancillary service, which opens up many opportunities for different actors of power system. This reactive power is essential to cope up for the inductive capacitive effects of the long transmission lines, and due to the varying nature of the loads. The reactive power management leads to voltage stability, and thus the paper deals with the review of the most common procedures for the compensation of reactive power. The paper then develops a model for a coal-fired power plant, and then compares the performance of different compensation techniques in terms of costs, efficiency in terms of real power losses, and performance capability in terms of response time and amount of reactive power. The paper then realizes the trade-off between different techniques, and then an aggregation of different techniques are used to strategize the coordinated control of techniques with respect to the requirements. Finally, a topology of this coordinated control mechanism is developed in accordance with the Italian regulations.
\end{abstract}

Keywords-reactive power mismatches; voltage stability; aggregators; DIgSILENT; coordinated control; Italian regulations; flexibility for power plants; TSO

\section{INTRODUCTION}

Distributed energy resources, and the variable demand at load side put the distribution system reactive power uncertain, and these uncertainties add up with the inductive and capacitive nature of the long transmission network. Voltage loading and voltage stability are the key parameters of concern, and it requires actions for TSO to put the overall grid in stable mode. Importance of reactive power is discussed in [1] [2], and the mismatches due to transmission line is explained in [3].

Apart from transmission lines, the other major problem creator is the varying nature of load-side demand. Distributed energy resources directly influence this demand, as the system operators have low visibility on their prevalence as in [4]. These cause different power system reliability and stability issues as in [5] [6] [7], where the key parameter of interest here is voltage stability which is achieved through balancing reactive power throughout the grid. It is therefore the responsibility of TSO to maintain the balance, which in turn asks for the services from generators (either as mandatory provision [8] [9], or with remuneration, or compensation [10]).

European Union's Horizon 2020 research and innovation programme under the Marie Sklodowska-Curie grant agreement No. 676042.
The paper is organized in the following sections: Section II discusses the different techniques for the reactive power provision. Section III demonstrates the model for the coal-fired power plant, and the model of the different compensation techniques in DIgSILENT (Figure 1). It also presents a test case for the requirement of reactive power by TSO. Section IV evaluates the performance of different techniques for the defined test case. Section $\mathrm{V}$ evaluates the performance of aggregation of the different techniques for the test case, and a strategy for this aggregated control is presented. Section VI concludes the paper

\section{COMPARISON OF REACTIVE POWER COMPENSATION TECHNIQUES}

Different reactive power compensation techniques are available in the current market scenario. For the generating system mismatches, AVR (Automatic Voltage Regulators) [11] and PSS (Power System Stabilizer) [11] [12] are enough. A variety of voltage stability techniques are employed at distribution level, under volt/var control as in [13] [14] [15]. Most of the mismatches occur at the transmission level, and there are variety of practices for the solutions.

Use of synchronous generators is the most common way for the mismatches [16]. However, they have some limitations as presented in [17]. Another technique is the capacitor bank; however, they have the issue of non-linear voltage relationship. Synchronous condenser is another medium, which is designated for serving reactive power only as in [18]. Use of FACTS (Flexible AC Transmission Systems [19]) devices is another existing solution.

Two potential FACTS devices are SVC (Static VAR Compensator) and STATCOM (Static Synchronous Compensator) [20]. They differ in terms of their power electronics based controlling as either voltage, or current. Inverters within existing wind and solar plants are another sources [21], however their limitations are in terms of the amount of provision [22]. A detailed comparison for all these compensation devices is available in [23]. Table. I gives a comparison summary of different reactive power compensation techniques in terms of costs, loss of real power for the reactive power service, and the capability. 




Fig. 1. Architecture of the Model with the Compensation Devices, and the TSO Test Case

\section{MODELING OF THE SYSTEM AND THE DEFINITION OF TEST-CASE}

The coal-fired power plant is selected as the generation medium, which has the capability to provide up to I MW. Mechanical characteristics, design, profile, and the layout for the power plant are followed from [24]. DIgSILENT only models the output electrical node.

The idea is to connect the compensation devices in section II (one by one) to the point of interconnection at the transmission and generation networks. Then, the same point of interconnection is at the TSO controller node; and it can be used to test the reactive power test case scenario developed by TSO. The details of the test case are elaborated in the following paragraph, but the main reactive power requirements by TSO can be taken as the parameter of interest. The model is represented in Figure 1.

The test case is the reactive power demand, by TSO, of up to 40 MVAR, with 20 MVAR inductive and 20 MVAR capacitive. In other words, the TSO has the responsibility to manage both provision and absorption of reactive power capacity of 20 MVAR. It is obvious that the TSO goes to no-provision mode, in case of no needs. In usual scenario, the TSO asks for the provision from conventional power plants, as synchronous generators. However, in this experimented test case, other compensators also contribute on top of these power plants, at the point of interconnection.

The D-STACOM is used, with current control functionality as in [25], with the capability of 20 MVAR. The DIgSILENT model for this STATCOM is used from [26]. The STATCOM is connected to the low-voltage end of the transformer to assure the voltage compatibility, and the high voltage end is supplied to the point of interconnection for reactive power provision. For the SVC, the model and controller are both complied with [27]. DIgSILENT model [28], part II, is used as reference.
The models for synchronous generator is available in DIgSILENT under synchronous machine, with the option to select generator. The same model is taken for synchronous condenser, with the possibility to put active power as zero, in the load flow data. The same is the case with capacitors, connected in shunt orientation. Inverters are extracted as part of the PV panel model of DIgSILENT.

For each of three scenarios by TSO, described earlier, the compensation devices are added simultaneously to the existing POI in DIgSILENT, and then the results are analyzed in terms of the parameters defined in Table. I. The results are discussed in the proceeding section.

TABLE I.

Reactive Power Compensation Techniques

\begin{tabular}{|l|l|c|c|}
\hline \multirow{2}{*}{$\begin{array}{c}\text { Source of } \\
\text { Re-active } \\
\text { Power }\end{array}$} & $\begin{array}{c}\text { PAPEX-OPEX (Capital } \\
\text { and Operating Costs) }\end{array}$ & $\begin{array}{c}\text { Amount of } \\
\text { Reactive } \\
\text { Power } \\
\text { Provision }\end{array}$ & $\begin{array}{c}\text { Loss of } \\
\text { Real } \\
\text { Power } \\
\text { Efficiency }\end{array}$ \\
\cline { 2 - 4 } $\begin{array}{l}\text { Synchronous } \\
\text { Generator }\end{array}$ & Very High - High & High & Very Low \\
\hline $\begin{array}{l}\text { Synchronous } \\
\text { Condensor }\end{array}$ & High - High & High & Very Low \\
\hline $\begin{array}{l}\text { STATCOM } \\
\text { SVC }\end{array}$ & Very High - Low & Medium & High \\
\hline $\begin{array}{l}\text { Switched } \\
\text { Capacitors }\end{array}$ & Medium - Very Low & Low & Low \\
\hline $\begin{array}{l}\text { Inverters (For } \\
\text { Already } \\
\text { Installed } \\
\text { Renewable } \\
\text { Resources } \\
\text { Plants) }\end{array}$ & Medium - Low & Very Low \\
\hline
\end{tabular}


TABLE II

Aggregation Method

\begin{tabular}{|c|c|c|c|}
\hline \multirow{2}{*}{$\begin{array}{c}\text { Serial } \\
\text { Number }\end{array}$} & \multicolumn{3}{|c|}{ Parameters for Aggregation } \\
\hline & Base Technique & Aggregated Technique & $\begin{array}{l}\text { Zone of } \\
\text { Interest }\end{array}$ \\
\hline 1 & $\begin{array}{l}\text { Synchronous } \\
\text { Generator }\end{array}$ & STATCOM & $\begin{array}{l}\text { Transient } \\
\text { response }\end{array}$ \\
\hline 2 & $\begin{array}{l}\text { Synchronous } \\
\text { Generator }\end{array}$ & $\begin{array}{l}\text { Synchronous } \\
\text { Condenser }\end{array}$ & $\begin{array}{c}\text { High } \\
\text { efficiency } \\
\text { and steady } \\
\text { state } \\
\text { response }\end{array}$ \\
\hline 3 & $\begin{array}{l}\text { Synchronous } \\
\text { Generator }\end{array}$ & SVC and Capacitors & $\begin{array}{l}\text { Cost (in } \\
\text { terms of } \\
\text { invest- } \\
\text { ment as } \\
\text { CAPEX } \\
\text { and } \\
\text { OPEX) }\end{array}$ \\
\hline 4 & $\begin{array}{l}\text { Synchronous } \\
\text { Generator }\end{array}$ & Inverters & $\begin{array}{l}\text { Cost (in } \\
\text { terms of } \\
\text { invest- } \\
\text { ment as } \\
\text { CAPEX } \\
\text { and } \\
\text { OPEX) }\end{array}$ \\
\hline
\end{tabular}

\section{COMPARISON AMONGST DIFFERENT TECHNIQUES FOR FLEXIBILITY PROVISION}

All the mentioned components are added at the POI, and investigated in DIgSILENT. The results from DIgSILENT indicate the trade-off in terms of the performance of each of the respective compensation device. STATCOM provides the best response in terms of voltage regulation, and transient response. This faster response is beneficial for TSO, in case of urgent needs for the restoration services.

However, synchronous generators on the other hand provides the maximum amount of provision, with a very high efficiency in terms of real power losses. This is the ideal behavior during steady-state conditions. However, the provision is only at the stake of slow response. Synchronous condenses almost poses the same characteristics, with the fact that the provision is higher as there is no real power requirement. However, the hurdles are the times and costs related to the conversion of synchronous machines for the purpose.

SVC has higher efficiency than STATCOM, in terms of fewer losses. However, the non-linear voltage control for reactive power makes it useful only for distribution systems. Hence, the solution is not very recommended for TSO and large power plants. The reason is the same behind the elimination of capacitor banks.

Inverters already installed in the wind and solar plants, are also evaluated in the comparison. However, there are issues of synchronizing them with the grid, and they can only provide a very small portion of reactive power, which does not comply with the requirements of TSO and large power plants.

Based on the analysis of these results, the techniques are aggregated on the basis of weighted combinations of transient and steady state characteristics, costs, amount of provision, and the efficiency. Next section is dedicated for this purpose.

\section{COMPARISON AMONGST DIFFERENT AGGREGATED TECHNIQUES FOR FLEXIBILITY PROVISION}

Based on the results of previous section, each technique is characterized in terms of five factors: steady state response, transient response, cost for service, amount of reactive power it can provide, and the loss of efficiency due to the provision. Each factor is rated in a scale of 0 to 4 (in increments of 1 ) for each technique. Then the techniques are aggregated one by one in the fashion described in Table. II.

According to Italian regulations, distributed generators, customers, and small third party providers cannot participate in TSO services market. TSO only asks for these services from power plants, as mandatory provision. Due to these restrictions, all the aggregations are performed on top of conventional synchronous generators in Table. II. STATCOM is added to ensure better performance in terms of transient conditions. This makes it technically the best solution.

Synchronous condensers on top of synchronous generators, give a very high steady-state provision and efficiency. This can be the most readable solution, but with some limitations discussed earlier. SVC and capacitor bank, together with these plants, make the best economical solution. However, the solution will not be feasible.

Inverters and converters within wind and solar panels can also provide economical solution, but the feasibility is in question again due to the low availability of reactive power. For explicit inverters, the capital costs would be very high. In addition, the topic is not in the context of this paper. Technique names are mentioned in Table. III. Ratings for each technique and their aggregated weightage is provided in Table. IV.

TABLE III.

Technique Listing

\begin{tabular}{|c|c|}
\hline $\begin{array}{c}\text { Technique } \\
\text { Number }\end{array}$ & Name of Technique \\
\hline 1 & Synchronous Generator \\
\hline 2 & Synchronous Condenser \\
\hline 3 & SVC \\
\hline 4 & Inverters within Farms \\
\hline 5 & $\begin{array}{c}\text { Synchronous Generator with } \\
\text { STATCOM }\end{array}$ \\
\hline 6 & $\begin{array}{c}\text { Synchronous Generator with } \\
\text { Synchronous Condenser }\end{array}$ \\
\hline 7 & $\begin{array}{c}\text { Synchronous Generator with } \\
\text { SVC }\end{array}$ \\
\hline 9 & $\begin{array}{c}\text { Synchronous Generator with } \\
\text { Inverter }\end{array}$ \\
\hline 10 & $\begin{array}{c}\text { Synchronous Generator with } \\
\text { STATCOM, and } \\
\text { Synchronous Condenser }\end{array}$ \\
\hline 11 & \\
\hline &
\end{tabular}


TABLE IV.

Weightage Lookup Criteria*

\begin{tabular}{|c|c|c|c|c|c|}
\hline \multirow{2}{*}{$\begin{array}{l}\text { Tech- } \\
\text { nique }\end{array}$} & \multicolumn{3}{|c|}{ Performance Criterion } & \multirow[b]{2}{*}{ Efficiency } & \multirow[b]{2}{*}{$\begin{array}{l}\text { Am- } \\
\text { ount }\end{array}$} \\
\hline & $\begin{array}{c}\text { Steady state } \\
\text { response }\end{array}$ & $\begin{array}{l}\text { Transient } \\
\text { response }\end{array}$ & Cost & & \\
\hline 1 & 4 & 2 & 1 & 4 & 4 \\
\hline 2 & 4 & 2 & 1 & 4 & 4 \\
\hline 3 & 2 & 4 & 2 & 1 & 3 \\
\hline 4 & 2 & 3 & 2 & 3 & 2 \\
\hline 5 & 0 & 3 & 3 & 4 & 2 \\
\hline 6 & 1 & 2 & 3 & 4 & 1 \\
\hline 7 & 6 & 6 & 3 & 5 & 7 \\
\hline 8 & 8 & 4 & 2 & 8 & 8 \\
\hline 9 & 6 & 5 & 3 & 7 & 6 \\
\hline 10 & 5 & 4 & 4 & 8 & 5 \\
\hline 11 & 10 & 8 & 4 & 9 & 11 \\
\hline
\end{tabular}

The aggregation is further performed amongst the four actors in Table. II; and then the optimal strategy is organized that produce the reactive power flexibility at optimal cost, and with optimal efficiency with an optimal steady state and transient response. These optimal strategies depend on the application areas for the TSO. From Table. IV, it is clear that technique 8 (synchronous generator with synchronous condenser) is best amongst 7-10; in terms of steady state response (weight of 8), and amount of reactive power provision (weight of 8 ). The same technique 8 has a tie with technique 10 (synchronous generator with inverter) for efficiency, however, the later solution is not feasible. Therefore, it gets the edge here too.

It is obvious that aggregation of synchronous generator with synchronous condenser gives the best results for steady state response, efficiency, and amount of provision. The next step is to involve transient sate response, and cost related benefits in to this aggregation. Techniques 5 and 6 are already dis-regarded as part of Italian regulations, and their infeasibilities. The only competition left is amongst technique 3 and 4, i.e. STATCOM and SVC.

From Table. IV, it is evident that STATCOM is better than SVC for transient response, and they have tie for cost benefits. Therefore, STATCOM is used for aggregation in technique 11. Another justification is the infeasible behavior of SVC for TSO and power plants based requirements, as discussed in the previous section. Technique 11 (aggregation of synchronous generator, synchronous condenser, and STATCOM) gives the optimal strategy for reactive power to the power plants.

\section{CONCLUSION AND FUTURE WORK}

Based on Table. IV, a strategic mechanism is developed, Figure 2, which clearly indicates the optimal performance for reactive power provision for each defined zone. Validation of the architecture is done through extending the model, with
Table. IV, part 11, in DIgSILENT. The mechanism can provide extended flexibility and benefits to generators for the service of TSO.

In Figure 2, TSO maintains the provision of reactive power from conventional power plants for the three purposes: fast provision to ensure contingencies and urgent scenarios, high amount to ensure more imbalances, and efficient provision for better utilization. Generators decide for this provision through the flexibility curve generated at their controllers. These power plants have the tendency to take services from either synchronous condensers, or STATCOM, or both. Number 1 in the Venn diagram indicates the sole performance of synchronous generators. Number 2 combines the performance of synchronous generators with synchronous condensers. Number 3 includes STATCOM on top of them.

The strategy is characterized in better way by the plot in Figure 2. It is divided into four regions of operation, as an aggregated coordinated control, which operates under the flexibility of power plants to TSO. Region 1 is the normal condition, which is the current usual solution. Region 2 works primarily for high demand conditions, where other characteristics are improved too as compared to region 1 .

Region 3 is the operation when a faster response is needed, and this is achieved through integrating STATCOM in any of the two defined regions. Irrespective of the amount of provision, the needs to satisfy unexpected demands (for example, heavy penetration of distributed energy resource at a feeder cause change in current, with the protection relay to lose it's dependability) are ensured.

Region 4 is an ideal solution, which has the potential to be adapted in the future. It provides the added flexibility in terms of advantages of high reactive power provision in both steady and transient states, with an optimal efficiency and costs. Design of the power plant controller and the optimization tools for the flexibility curve can be the potential addition in the future work. All these regions are accessible to both generators and TSO (via controller at power plant).

Aggregation criterion depends on the specific TSO applications for a specific region. The applications can be the usual current solution with a trade-off in provision of reactive power, and the costs. It can be the most expensive option, but with high readiness. It can be the cheapest solution, but with low applicability to power plants due to the insufficient amount for participation in the ancillary market. It can also be the potential future solution with the maximum reactive power reserves.

The strategy in this paper is opted for the generators, as the Italian regulations do not permit the distributed generators, DSO, and other aggregator zones to take part in this service provision. In addition, as there is no remuneration for generators by TSO, thus the flexibility is main business strategy for the power plants. Future work includes the addition of large commercial inverters, and storage systems in the architecture. It can also include the way virtual power plants can give flexibility, and the work may be extended to the TSO of other European countries. Another potential addition is the involvement of other ancillary services to ensure the coordinated control mechanism for their improvement. 

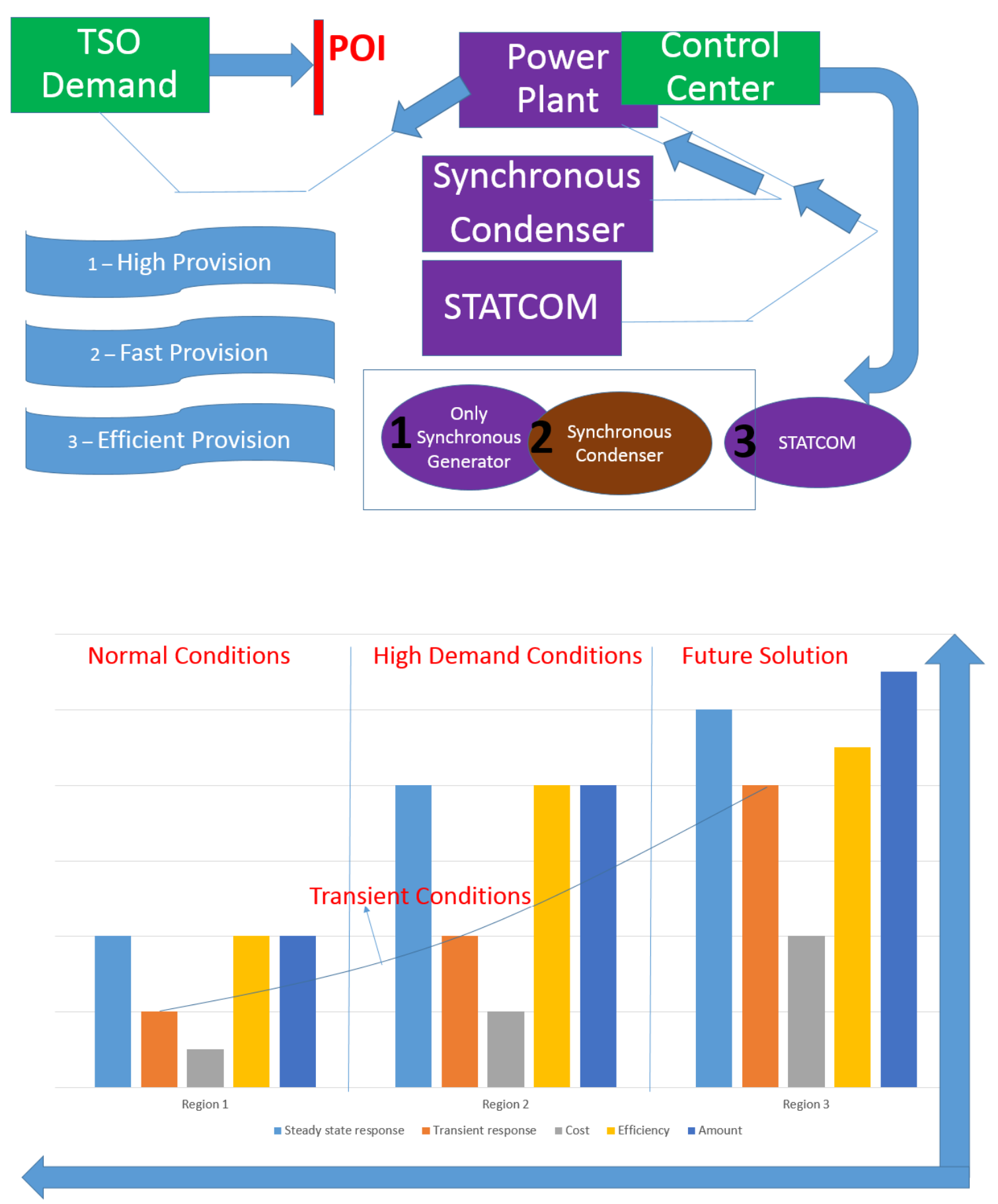

Fig. 2. Coordinated Control Mechanism 


\section{ACKNOWLEDGMENT}

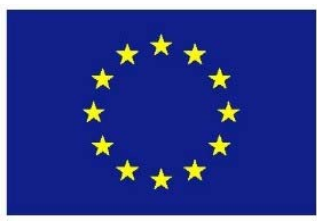

This project has received funding

from the European Union's Horizon 2020 research and innovation programme under the Marie Sklodowska-Curie grant agreement No 676042 .

\section{REFERENCES}

[1] P.W. Sauer, "What is reactive power?", UIUC, department of ECE. (https://pserc.wisc.edu/documents/publications/specialinterestpublicatio ns/gridreliability/)

[2] J. Parmar, "Importance of reactive power for system". (https://electricalnotes.wordpress.com/2011/03/21/importance-ofreactive-power-for-system/)

[3] H. Saadat, Power System Analysis, 3rd ed., USA: McGraw Hill, 2002.

[4] J. Smith, M. Rylander, L. Rogers, and R. Dugan, "It's all in the plans: maximizing the benefits and minimizing the impacts of ders in an integrated grid", IEEE Power and Energy Magazine, pp. 20-29, February 2015.

[5] Euramet,"SmartGrid-II", project. (http://gridmeas.eu/2017/05/15/newssmart-grids-ii-workshop-slides-now-available-online)

[6] Euramet,"FutureGrid", project. (http://futuregrid.emrp.eu)

[7] Euramet,"GridSens", project. (http://gridsens.eu)

[8] Number,"111-06", reference link. (https://www.arera.it/it/index.htm/)

[9] Grid refernce code,"Terna", reference link. (https://www.terna.it/en$\mathrm{gb} /$ sistemaelettrico/codicedirete.asp $x$ )

[10] M. Wolter, and B. Huhnerbein, "Identification of cross-border power flows in integrated networks based on the principle of superposition," IEEE Power and Energy Conference PECon, Malaysia, December 2008.

[11] P. Sivakumar, and C. Birindha, "Stability enhancement of dg sourced power system with modified avr and pss," International Conference on Computation of Power, Energy, Information and Communication ICCPEIC , India, April 2013.

[12] M. Shafiullah, M. J. Rana, and L. S. Coelho, "Power system stability enhancement by designing optimal pss employing backtracking search algorithm," International Conference on Clean Electrical Power, ICCEP , Italy, June 2017.

[13] A. M. Howlader, S. Sadoyama, and L. R. Roose, "Distributed voltage control method using volt-var control curve of photovoltaic inverter for a smart power grid system ," IEEE International Conference on Power Electronics and Drive Systems, PEDS , USA, December 2017.

[14] M. Rashidi, A. B. Ahmed, and A. Nasiri, "Application of a multi-port solid state transformer for volt-var control in distribution systems ," IEEE Power and Energy Society General Meeting, USA, July 2017.
[15] P. Raghavendra, and D. N. Gaonkar, "Coordinated volt-var control: online voltage-profile estimation in smart distribution networks," IEEE Industry Applications Magazine, vol. 24, pp. 14-22, January 2018.

[16] B. Zhou, W. Yao, T. Xu, Y. Dong, and X. Xie, "Investigating the influence of types and parameters of excitation systems on the dynamic reactive power reserve of synchronous generators ," International Conference on Fuzzy Systems and Knowledge Discovery , FSKD, China, August 2015.

[17] M. M. Adibi, and D. P. Milanicz, "Reactive capability limitation of synchronous machines," IEEE Transactions on Power Systems, vol. 9, pp. 29-40, February 1994.

[18] F. O. Igbinovia, G. Fandi, Z. Muller, J. Svec, and J. Tlusty, "Cost implication and reactive power generating potential of the synchronous condenser ," International Conference on Intelligent Green Building and Smart Grid, IGBSG, Czech Republic, June 2016.

[19] N. G. Hingorani, "FACTS- flexible ac transmission system," International Conference on AC and DC Power Transmission, UK, September 1991.

[20] N. G. Hingorani, and L. Gyugyi, "Understanding facts: concepts and technology of flexible ac trnasmission systems," Wiley: IEEE press, 2000.

[21] FERC report ,"Payment for reactive power", AD14-7, 2014.

[22] A. Ellis, R. Nelson, E. V. Engeln, R. Walling, J. MacDowell, L. Casey, E. Seymour, W. Peter, C. Barker, B. Kirby, and J. R. Williams, "Reactive power performance requirements for wind and solar plants, " IEEE Power and Energy Society General Meeting, USA, July 2012.

[23] J. Ali, S. Massucco, and G. Petretto, "Reactive power provision to tso-dso by aggregators and conventional generators," IEEE International Conference on Smart Grid Communications, SmartGridComm , Germany, October 2017.

[24] W. Pingyang, "Power systems and power plant control," Symposium, IFAC, China, 1986.

[25] J. I. Y. Ota, Y. Shibano, N. Niimura, and H. Akagi, “A phase-shifted-pwm d-statcom using a modular multilevel cascade converter ssbc - part I modeling, analysis, and design of current control," IEEE Transactions on Industry Applications , vol. 51, pp. 279-288, Mayy 2014.

[26] Open Source,"Do you have a model for a statcom", reference link. (https://www.digsilent.de/en/faq-reader-powerfactory/do-you-have-amodel-for-a-statcom-2.html)

[27] D. Jovcic, "Svc dynamic analytical model," IEEE Power Engineering Society General Meeting , Canada, July 2003.

[28] Open Source,"Do you have an example for modelling a svs-svc", reference-link.(https://www.digsilent.de/en/faq-readerpowerfactory/would-you-have-an-example-for-modelling-a-svs-svcstatic-var-system-compensator/searchfaq/SVC.html) 